Presented at the Eleventh Winter Workshop on Nuclear Dynamics, Key West, Florida, February 11 - 17, 1995, and to be published in the Proceedings

\title{
Progress in Collective Flow Studies From the Onset to Bevalac/SIS
}

\author{
M.A. Lisa \\ Lawrence Berkeley Laboratory \\ University of California, Berkeley, CA 94720
}

March 1995

This work was supported by the Director, Office of Energy Research, Division of Nuclear Physics of the Office of High Energy and Nuclear Physics of the U.S. Department of Energy under Contract DE-AC03-76SF00098 


\section{DISCLAIMER}

This report was prepared as an account of work sponsored by an agency of the United States Government. Neither the United States Government nor any agency thereof, nor any of their employees, make any warranty, express or implied, or assumes any legal liability or responsibility for the accuracy, completeness, or usefulness of any information, apparatus, product, or process disclosed, or represents that its use would not infringe privately owned rights. Reference herein to any specific commercial product, process, or service by trade name, trademark, manufacturer, or otherwise does not necessarily constitute or imply its endorsement, recommendation, or favoring by the United States Government or any agency thereof. The views and opinions of authors expressed herein do not necessarily state or reflect those of the United States Government or any agency thereof. 


\section{DISCLAIMER}

Portions of this document may be illegible in electronic image products. Images are produced from the best available original document. 


\title{
Progress in Collective Flow Studies From the Onset to Bevalac/SIS
}

\author{
M. A. Lisa \\ Nuclear Science Division, Lawrence Berkeley Laboratory \\ University of California, Berkeley, CA 94720, USA
}

\begin{abstract}
Collective flow in heavy ion collisions was first observed experimentally more than a decade ago at the Bevalac by the Plastic Ball collaboration. Although early calculations had suggested that measurement of the flow would place tight constraints on the nuclear equation of state, uncertainties in other input parameters of microscopic models, which also affect the flow, led to large ambiguities in the equation of state. This talk will discuss recent flow studies that attempt to overcome these difficulties. The EOS and FOPI experiments at the Bevalac and SIS accelerators have measured flow in the 200-2000 A.MeV bombarding energy range with better acceptance, particle identification, and systematics than was previously available. Meanwhile, programs at MSU and GANIL are studying the disappearance of flow around $50 \mathrm{~A} \cdot \mathrm{MeV}$. Systematic comparison of these data with predictions of microscopic models is beginning to reduce the ambiguities in the extraction of physics quantities. Also, new directions in flow studies, such as the flow of produced particles and radial flow, offer the possibility of further information from flow studies. Recent accomplishments and new directions in flow studies are discussed, and areas where further study is needed are pointed out.
\end{abstract}

\section{Introduction}

Early hydrodynamical models of heavy ion collisions predicted the existence of a strong collective component, called flow, in the emission pattern of reaction products (see [1] and references therein). These models suggested that the strength of nuclear flow is strongly dependent on the nuclear compressibility, raising the hope that the nuclear equation of state (EoS) could be mapped out through flow measurements $[1,2]$. Reaction models, such as the internuclear cascade [3], which lacked an explicit nuclear EoS, predicted little or no flow [4].

Directed flow was first identified experimentally [5] in heavy ion collisions at the Bevalac more than 10 years ago. Comparative studies (see, e.g. [6]) of early sidewards flow measurements with theoretical predictions proved unable to extract definitively the nuclear EoS. Ambiguities arose from both experimental and theoretical uncertainty. Experimentally, limited acceptance and particle identification (PID) capabilities hampered systematic comparison with model predictions [7]. On the theoretical side, parameters in the models, other than the EoS, were found to have a strong effect on flow [8]. The effect on the flow of varying "physics" inputs to models, such as 


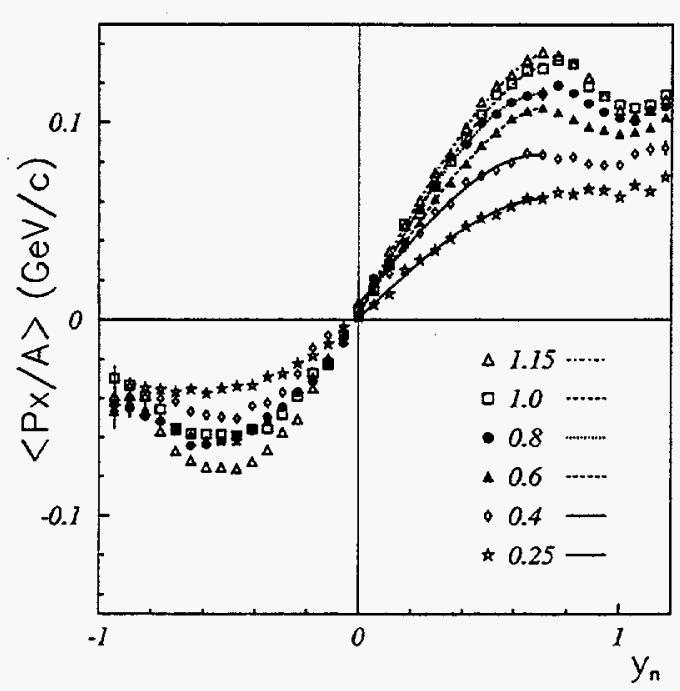

Fig. 1: "S"-shaped flow curves measured for semi-central Au+Au collisions at the Bevalac by the EOS collaboration. From [20].

momentum-dependent terms in the mean field $[9,10]$, the in-medium nucleon-nucleon cross section $\sigma_{n n}$ [11], and fragment formation mechanisms [12] has been studied. Also, the effects of more "technical" details of the models have been studied, such as energy and angular momentum conservation $[10,13]$, realistic treatment of the nuclear surface [14] and binding energy [4], and effects of the nucleon "uncertainty principle" [15]. It became increasingly clear that flow measurements with better acceptance, accuracy, and systematics were needed, if the subtle effect of the EoS were to be teased out of the data through comprehensive comparison to theory.

In the past decade, a large subfield has developed around flow studies, and much has been learned. New aspects of flow, such as the squeeze-out and radial flow, have been identified, and the systematics of flow are being mapped out over a large energy range. New $4 \pi$ heavy ion detectors $[16,17,18]$, designed to overcome the shortcomings of the Plastic Ball and Streamer Chamber, are providing a much clearer and complete view of the flow phenomenon. Furthermore, more realistic dynamical models are now in place that allow more direct comparison with experiment. Some recent developments in flow studies at bombarding energies in the range $\mathrm{E} \approx 10-2000 \mathrm{~A} \cdot \mathrm{MeV}$ are discussed here.

\section{Repulsive Sideward Flow}

Repulsive sidewards flow is characterized by a non-isotropic emission pattern, in which particles emitted forward and backward in the c.m. are preferentially found in the reaction plane on opposite sides of the beam direction. Sidewards flow is commonly measured with Transverse Momentum Analysis [19], in which the average momentum per nucleon in the reaction plane is plotted as a function of c.m. rapidity. The slope of the resulting " $\mathrm{S}$ "-shaped curve at $y_{c . m .}=0$ is taken as a measure of the collective motion, and is often simply called "flow." Figure 1 shows flow curves recently measured by the EOS collaboration for $\mathrm{Au}+\mathrm{Au}$ at $\mathrm{E} / \mathrm{A}=0.25-1.15 \mathrm{GeV}$ [20].

It had been suggested that the flow of composite fragments might be more sensitive to the EoS than proton flow or inclusive flow measurements [12]. With good PID, the 


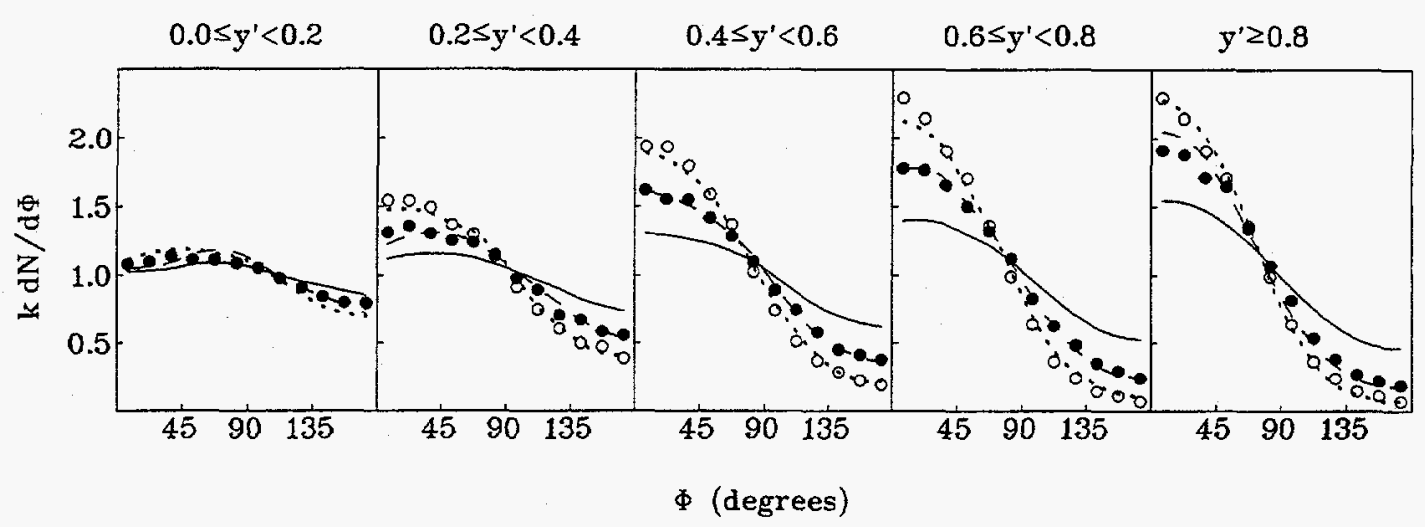

Fig. 2: Azimuthal distributions with respect to the reaction plane for protons (solid line), deuterons (filled points), and $A=3$ fragments (open points) are shown, as well as the square of the proton distribution (dashed line), and the deuteron distribution taken to the power of 3/2. From [21].

EOS TPC can study flow for the first time seperately for different particle species. These detailed data have been used to construct more stringent tests of dynamical models than had previously been possible [20].

An attempt to understand the flow mass systematics themselves - independent of a dynamical model - has recently been undertaken [21]; some results are shown in Figure 2. Here, the azimuthal distribution with respect to the reaction plane is shown for protons, deuterons, and $A=3$ particles in 5 rapidity windows in the c.m. frame. Directed flow manifests itself in a tighter focussing in the reaction plane for particles at forward rapidity, and the mass dependence of flow [20] is shown by a tighter focussing of the heavier particles. When a low $p_{t}$ is applied to the data, one observes that a simple coalescence prescription is able to reproduce the mass dependence of flow remarkably well-squaring (cubing) the proton azimuthal distribution reproduces the deuteron $(A=3)$ distribution [21]. Thus, a clearer and more complete understanding of some flow systematics is emerging from the new flow studies. Further, this new finding validates the use of one-body models that lack composite cluster formation, such as the BUU, in the study of flow physics [6].

Recently, predictions of a BUU model have been compared to two data sets in an attempt to reduce the ambiguity in determining the EoS from flow measuremnents [24]. Figure 3 shows measured flow as a function of event centrality by the Plastic Ball collaboration for the symmetric system $\mathrm{Nb}+\mathrm{Nb}$ [22], and by the Diogene collaboration for the asymetric system $\mathrm{Ar}+\mathrm{Pb}[23]$, both at $\mathrm{E} / \mathrm{A}=400 \mathrm{~A} \cdot \mathrm{MeV}$. BUU flow predictions using four parametrizations of the EoS are compared to the measurements. It is seen that only a soft EoS with momentum dependence interactions (MDI) in the mean field reproduces both data sets, whereas comparison with either one of the measurements alone could not uniquely identify the correct EoS. This would leave the largest uncertainty in the model associated with the in-medium cross section $\sigma_{n n}$. It has been suggested that flow sytematics as a function of system mass may reduce this ambiguity as well. Thus, there is hope that, upon comparison with the more complete data sets now coming from the new flow measurements, dynamical models 

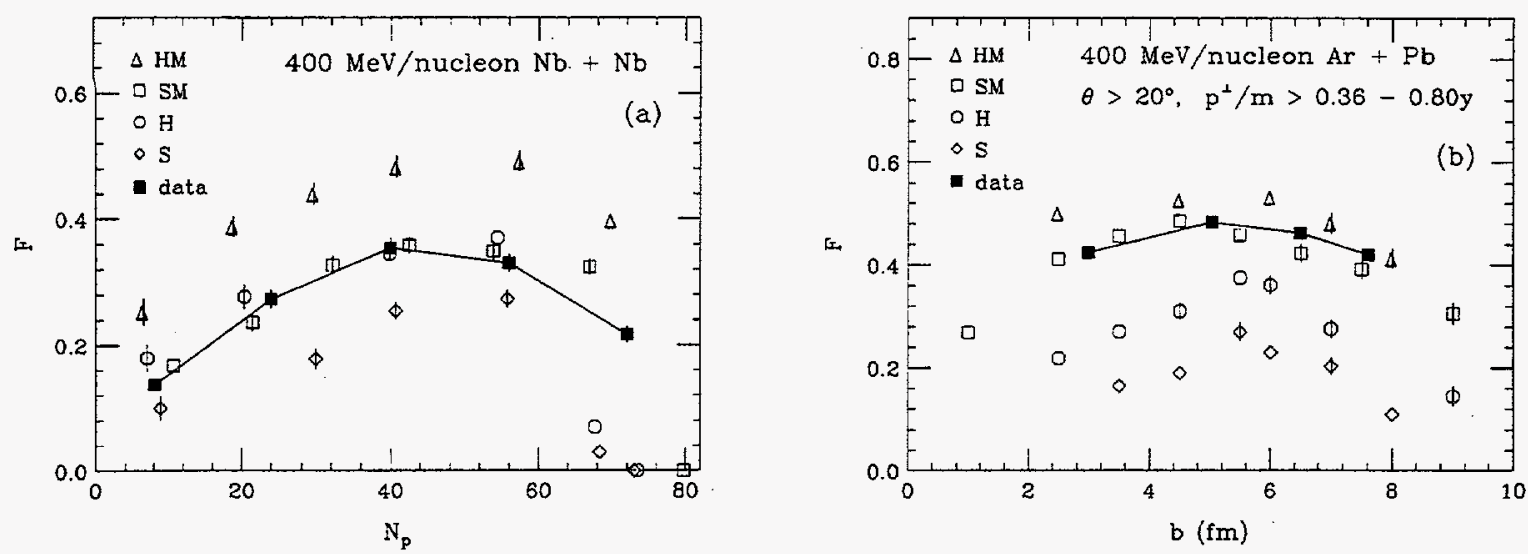

Fig. 3: Flow as a function of event centrality is shown for $\mathrm{Nb}+\mathrm{Nb}$ [22] and $\mathrm{Ar}+\mathrm{Pb}$ [23] at 400 $\mathrm{A} \cdot \mathrm{MeV}$. Also shown are predictions of a BUU model with soft and hard EoS, with and without momentum dependence in the mean field. From [24].

may yet provide reliable information on the nuclear EoS.

A new direction in the study of collective decay modes looks at the flow of produced particles. With VUU and QMD model calculations Bass and collaborators [25,26] have explored $\pi$ flow in heavy ion collisions. For all but the most central collisions, pions are predicted to "anti-flow" - that is, flow in the reaction plane in the direction opposite the direction of nucleon flow. Preliminary confirmation of this prediction has been seen in Au+Au collisions at $1 \mathrm{~A} \cdot \mathrm{GeV}$ by the FOPI collaboration [27]. The magnitude and sign of the pion flow for varying event centrality may depend strongly on the nuclear EoS [26], providing another sensitive aspect of the flow phenomenon. Analysis of $\pi$ flow is in progress with the new data sets at LBL and GSI [28].

\section{The Disappearance of Flow}

The nuclear force is repulsive at Bevalac/SIS bombarding energy, leading to projectile fragment scattering to positive angles in the reaction plane, which we define as "positive flow." At lower bombarding energy, around $50 \mathrm{~A} \cdot \mathrm{MeV}$, the nuclear interaction is attractive, and one expects the projectile-target pair to partially rotate around each other before scattering to negative angles, leading to "negative flow." Flow disappears at the so-called balance energy, $E_{b a l}$, where these two effects cancel each other. The disappearance of flow was first observed in the La+La system [29], and has since been measured for a large number of target-projectile combinations from $\mathrm{C}+\mathrm{C}[30]$ to (a lower limit for) $\mathrm{Au}+\mathrm{Au}[31]$.

The mass dependence of the balance energy is shown in Figure 4 . The $A^{-1 / 3}$ dependence of $E_{b a l}$ can qualitatively be understood in terms of the competition between the repulsive effect of hard collisions between projectile and target nucleons (which scales as A), and the attractive interaction of the nuclear surfaces (which scales as $A^{2 / 3}$ ). Comparisons of measurements with predictions of a BUU model [30] show that $E_{b a l}$ is relatively insensitive to the EoS used in the BUU, but that there is a sensitivity 


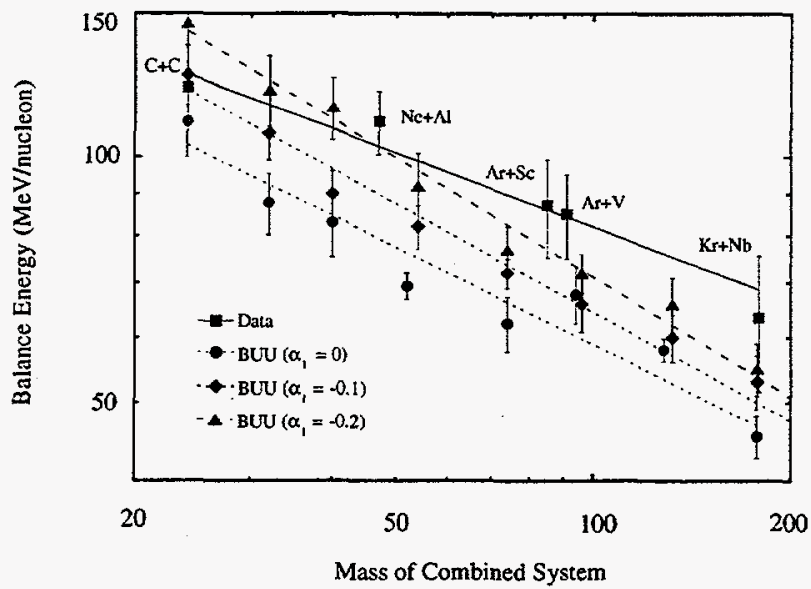

Fig. 4: The observed mass dependence of the disappearance of flow is compared to BUU predictions using a soft $\mathrm{E} O \mathrm{~S}$, and $\sigma_{n n}$ at nuclear matter density reduced by $0 \%, 10 \%$, and $20 \%$ from its free value. Lines guide the eye. From [30].

to the in-medium $\sigma_{n n}$. In particular, as shown in Figure 4, the model reproduces the data best if $\sigma_{n n}$ at nuclear matter density is reduced by $20 \%$ compared to its free value. Thus, flow studies at these low energies may provide a handle on one of the main physics inputs to models.

A potential source of ambiguity in extracting physics from comparisons with the BUU model, however, arises from the fact that the treatment of the nuclear surface is not well defined in BUU models. The details of the treatment of the nuclear surface in the model can strongly affect the predicted value of $E_{b a l}$ [14]. One way to probe the details of the nuclear surface on flow may be to to measure the impact parameter dependence of the disappearance of flow [32]. So far, this measurement has been done in few systems only [33], but the systematics are currently being extended [34].

\section{Squeeze-out}

Although I have no time to discuss this mode in detail, another collective mode observed in particle emission patterns is the so-called "squeeze-out" effect [35], corresponding to a preference for particles to be emitted perpendicular to the reaction plane. Dynamical models indicate that the squeeze-out magnitude in heavy ion collisions may be quite sensitive to the nuclear EoS [36]. However, for the early measurements, the effects of detector acceptance are of similar magnitude as the change in squeeze-out using different equations of state [36].

The squeeze-out effect is being measured by new detector systems with better and simpler acceptance for protons and neutrons [37], as well as for charged and neutral pions $[38,39]$. It will be exciting to see what insights are gained by comparison with model predictions. 


\section{Radial Flow}

Besides the directed modes of collective motion - squeeze-out and attractive and repulsive flow - there is another possible collective mode in the decay of an excited nuclear system - isotropic radial flow. In the simple thermodynamic "fireball" model [40], one assumes a non-isentropic expansion resulting in transverse momentum spectra that are, in the classical limit, of Maxwell-Boltzmann type, the inverse exponential slope representing the source temperature $\mathrm{T}$. If, on the other hand, the decay resembles more hydrodynamic expansion, due to a short mean free path of escaping nuclear matter, one would expect a "shoulder arm" on the spectra resulting from the ordered radial motion, or "blast wave." The shape of the spectra then would be dictated by the radial flow velocity $\beta$ and the intrinsic temperature (that is, in the flow frame, not the overall source frame) of the source. Such a scenario was considered to explain proton and pion spectra at $90^{\circ}$ in the $\mathrm{Ne}+\mathrm{NaF}$ reaction at $800 \mathrm{~A} \cdot \mathrm{MeV}[41]$. However, only with the availability of the recent high quality data sets have systematic studies of the radial flow in heavy ion collisons been possible.

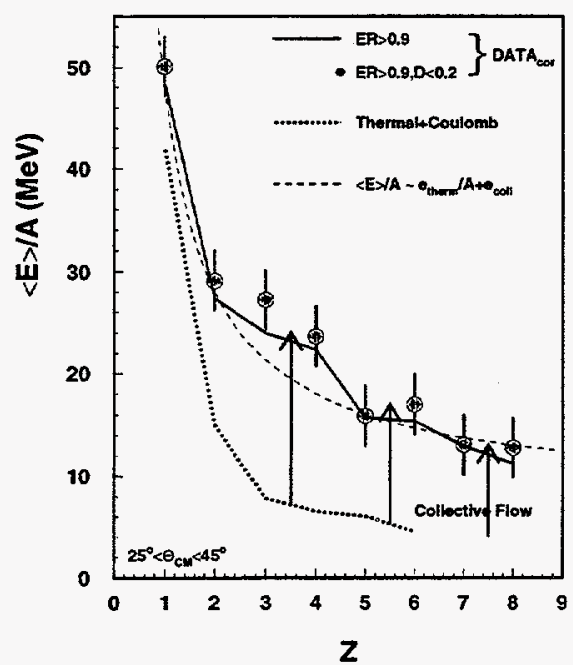

Fig. 5: The average energy per nucleon of fragments emitted from central $\mathrm{Au}+\mathrm{Au}$ collisions at $\mathrm{E}=150 \mathrm{~A} \cdot \mathrm{MeV}$ is plotted as a function of the fragment charge. Average energy values expected from thermal and Coulomb effects alone, indicated by the dotted line, fall below the observed values. The effects of radial flow are indicated by the arrows, and bring the average energies to the dashed line. From [42].

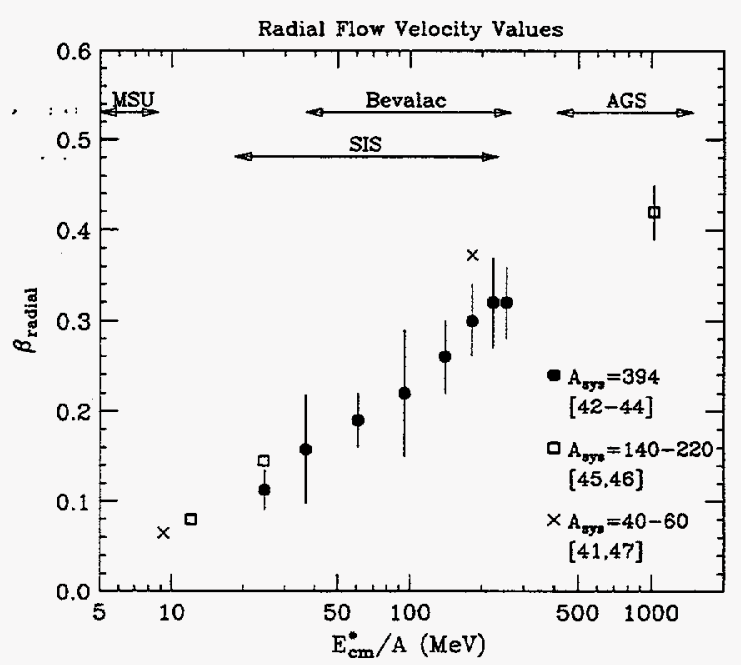

Fig. 6: Radial flow velocities extracted from heavy ion measurements as a function of the available energy in the center of mass. Flow values for various size systems (projectile target combinations) are indicated by different marker types. For each measurement reported, the average flow velocity is plotted. References indicated in figure.

A systematic study of $\mathrm{Au}+\mathrm{Au}$ reactions at $150 \mathrm{~A} \cdot \mathrm{MeV}$ [42] reveals a strong isotropic collective component to the energy of fragments emitted from central collisions. If there were no radial flow, the average energy of a fragment would be proportional to the source temperature and independent of its mass (a Coulomb barrier adds a small additional component proportional to the fragment charge). As is 
shown in Figure 5, especially for the heavier fragments, the average fragment energy in central collisions is too high to be explained by thermal and Coulomb effects alone. The energies are better reproduced if one assumes the presence of a radial flow which takes $30-50 \%$ of the available energy. Studies of energy spectra shapes for Au+Au collisions at other bombarding energies [43,44] also indicate the presence of radial flow that accounts for about half of the available energy in the c.m. system. Suprisingly (unfortunately?), model calculations suggest that the strength of the radial flow is quite insensitive to the EoS used in the model [44]. Figure 6 summarizes most radial flow measurements published to date. The radial flow phenomenon appears to be a general feature of heavy ion collisions, not restricted to a given energy range.

The cause of the radial flow in heavy ion collisions deserves theoretical investigation, in light of the apparent insensitivity of predicted radial flow values to model parameters. However, the effects of having $50 \%$ of the available energy tied up in radial flow are of equal interest. These effects may include reduced entropy [48] and temperature of the participant source, and significant effects on fragment yields [49].

\section{Conclusions}

More than ten years after its discovery, the study of collective flow in heavy ion collisions continues as an active and productive area of research. Today, more sensitive and complete flow measurements challenge more sophisticated theoretical models. Here, a summary of some recent achievements and new directions in flow studies is given, followed by suggestions where future work should be devoted.

In the Bevalac/SIS energy range, a clearer understanding of the systematics of repulsive flow is emerging. These systematics are being used to stringently test microscopic reaction models. Ambiguities that had plagued early attempts to extract the nuclear EoS from flow measurements are being reduced through systematic model comparisons. In particular, varying the system asymmetry may disentangle the effects of the momentum and density dependence of the EoS, while the system size dependence of flow may hold independent information about the contribution from hard nucleon-nucleon collisions. Current investigations into the flow of produced particles holds great potential to yield physical insights into the EoS and particle transport properties.

At lower bombarding energies, the balance energy has been measured over a large mass range for symmetric systems, and evidence for a reduced in-medium $\sigma_{n n}$ is obtained through comparisons with BUU predictions.

Clear observations have now been made over a large range of bombarding enegies of a new mode of collective flow: isotropic radial flow, which accounts for about $50 \%$ of the available energy for participant nucleons in central collisions. We now see in the literature initial investigations into the effects of radial flow on other physical quantities. Such studies may cause us to review in a new light old questions about participant matter decay. 
All of these studies have led to greater refinement and sophistication of dynamical reaction models. Initially, this had the frustrating result of revealing ambiguities in EoS studies, as it became clear that other effects could modify the flow signal significantly. However, as the interplay between these effects becomes better understood, we find that flow studies can provide information about a range of physical processes, and hope again arises that the original goal of constraining the nuclear EoS may be in sight.

Further work needs to be done in flow studies. Directed flow effects have been observed at the AGS already [50]. The flow excitation function should be mapped out in the energy range spanning Bevalac/SIS energies, up to the maximum AGS energy, since the increasing importance of resonances in hot nuclear matter may affect the EoS. A measure of the evolution of the EoS with bombarding energy (temperature) may be important when considering the effect of collective motion at higher energyeven at CERN and RHIC. Another high priority should be measuring the flow excitation function in the energy range from the balance energy up to Bevalac/SIS energies. It is in this range that flow changes most rapidly as a function of energy, and here that hydrodynamic scaling is most strongly violated [51]. In the spirit of the study by Pan and Danielewicz [24], it may be worthwhile to study balance energy systematics for assymetric systems, to investigate possible effects of momentum dependent interactions at low energy. As mentioned above, the impact parameter dependence of $E_{b a l}$ should be measured, to reduce ambiguities associated with model treatment of the nuclear surface. Finally, much of our justification for investigating the nuclear EoS is derived from astrophysics, where one would like to understand neutron stars. Therefore, as radioactive beams become available, the collective aspects of nuclear reactions should be surveyed as a function of isospin.

Instructive conversations with Drs. P. Danielewicz, W.G. Lynch, G. Odyniec, A.M. Poskanzer, G. Rai, H.G. Ritter, and G.D. Westfall are gratefully appreciated. This work was supported in part by the Director, Office of Energy Research, Division of Nuclear Physics of the Office of High Energy and Nuclear Physics of the U.S. Department of Energy under contract DE-AC03-76SF0098.

\section{References}

[1] H. Stöcker and W. Greiner, Phys. Rep. 137, 227 (1986).

[2] The Nuclear Equation of State, W. Greiner and H. Stöcker, ed., NATO ASI Series B, Vol 216A (1989).

[3] J. Cugnon, T. Mizutani, and J. Vandermeulen, Nucl. Phys. A352, 505 (1981).

[4] J. Molitoris, et al., Phys. Rev. C33, 867 (1986).

[5] H.A. Gustafsson, et al., Phys. Rev. Lett. 52, 1590 (1984).

[6] B. Blättel, V. Koch, and U. Mosel, Rep. Prog. Phys. 56, 1 (1993).

[7] V. Koch, et al., Nucl. Phys. A532, 715 (1991).

[8] B. Blättel, et al., Phys. Rev. C43, 2728 (1991).

[9] J. Aichelin, et al., Phys. Rev. Lett. 58, 1926 (1987). 
[10] J. Zhang, S. Das Gupta, and C. Gale, Phys. Rev. C50, 1617 (1994).

[11] G.F. Bertsch, et al., Phys. Lett. B189, 384 (1987).

[12] G. Peilert, et al., Phys. Rev. C38, 1402 (1989).

[13] C. Gale and S. Das Gupta, Phys. Rev. C42, 1577 (1990).

[14] D. Klakow, G. Welke, W. Bauer, Phys. Rev. C48, 1982 (1993).

[15] J. Jänicke and J. Aichelin, Nucl. Phys. A547, 542 (1992).

[16] G. Rai, et al., IEEE Trans. Nucl. Sci. 37, 56 (1990).

[17] A. Gobbi et al., Nucl. Instrum. Meth. Phys. Res., Sect. A 324, 156 (1993).

[18] G.D. Westfall et al., Nucl. Instrum. Meth. Phys. Res., Sect. A 238, 347 (1985).

[19] P. Danielewicz and G. Odyniec, Phys. Lett. 157B, 146 (1985).

[20] M.D. Partlan, et al., preprint LBL-36280, submitted to Phys. Rev. Lett.

[21] S. Wang, et al., Phys. Rev. Lett., in press (1995).

[22] H.A. Gustafsson, et al., Mod. Phys. Lett. A3, 1323 (1988).

[23] J. Gosset, et al., in Ref. [2].

[24] Q. Pan and P. Danielewicz, Phys. Rev. Lett. 70, 2062 (1993).

[25] S.A. Bass, et al., Phys. Lett. B302, 381 (1993).

[26] S.A. Bass, et al., Phys. Rev. bf C51, R12 (1995).

[27] D. Pelte, W. Reisdorf, T. Wienold, and the FOPI collaboration, GSI 09-93.

[28] J. Kintner and T. Wienold, private communications.

[29] D. Krofcheck, et al., Phys. Rev. Lett. 63, 2028 (1989).

[30] G.D. Westfall, et al., Phys. Rev. Lett. 71, 1986 (1993).

[31] W.M. Zhang, et al., Phys. Rev. C42, R491 (1990).

[32] J.P. Sullivan, et al., Phys. Lett. B249, 8 (1990).

[33] J.C. Angelique, et al., contribution to XXXI Interational Winter Meeting on Nuclear Physics, Bormio, ed I. Iori

[34] R. Pak, contribution to this conference.

[35] H.H. Gutbrod, et al., Phys. Lett. B216, 267 (1989).

[36] C. Hartnack, et al., Mod. Phys. Lett. A9, 1151 (1994).

[37] D. Lambrecht, et al., Z. Phys. A350, 115 (1994).

[38] L.B. Venema, et al., Phys. Rev. Lett. 71, 835 (1993).

[39] D. Brill, et al., Phys. Rev. Lett. 71, 336 (1993).

[40] G.D. Westfall, et al., Phys. Rev. Lett. 37, 1202 (1976).

[41] P.J. Siemens and J.O. Rasmussen, Phys. Rev. Lett. 42, 880 (1979).

[42] S.C. Jeong, et al., Phys. Rev. Lett. 72, 3468 (1994).

[43] W.C. Hsi, et al., Phys. Rev. Lett. 73, 3367 (1994).

[44] M.A. Lisa, et al., preprint LBL-35504

[45] H.W. Barz, et al., Nucl. Phys. A531, 453 (1991).

[46] W. Bauer, et al., Phys. Rev. C47, R1838 (1993).

[47] D. Heuer, et al., Phys. Rev. C50, 1943 (1994).

[48] C. Kuhn, et al., Phys. Rev. C48, 1232 (1993).

[49] G.J. Kunde, et al., Phys. Rev. Lett. 74, 38 (1995), and contribution to this conference. 
[50] J. Barrette, et al., Phys. Rev. Lett. 73, 2532 (1994).

[51] A. Bonasera and L.P. Csernai, Phys. Rev. Lett. 59, 630 (1987), and contribution to this conference. 